\title{
Semicontinuity for representations of one-dimensional Cohen-Macaulay Rings
}

\author{
Y.A. Drozd* \\ Mechanics \& \\ Mathematics Faculty \\ Kiew University \\ Wladimirskaya 64 \\ 252017 Kiew \\ Ukraine
}

\author{
G.-M. Greuel** \\ Fachbereich Mathematik \\ Universität Kaiserslautern \\ Erwin-Schrödinger-Straße \\ 67663 Kaiserslautern \\ Federal Republic of Germany
}

October 30, 2018

\section{Contents}

$\begin{array}{ll}\text { Introduction } & 1\end{array}$

\begin{tabular}{lll}
\hline & Dense subalgebras & 3 \\
\hline
\end{tabular}

$\begin{array}{lll}2 & \text { Cohen-Macaulay Algebras } & 6\end{array}$

\begin{tabular}{lll}
\hline 3 Families of Modules & 8
\end{tabular}

\begin{tabular}{lll}
\hline 4 Families of algebras & 12
\end{tabular}

$\begin{array}{lll}5 \text { Some analogues } & 17\end{array}$

$\begin{array}{ll}\text { References } & 21\end{array}$

* Partially supported by the Foundation for Fundamental Research of the Ukraine and the Deutsche Forschungsgemeinschaft (DFG)

** Partially supported by the Deutsche Forschungsgemeinschaft (DFG) 


\section{Introduction}

"Algebraic families" of modules and algebras play an important role in several questions of representation theory. It is often especially useful to know that some "discrete invariants" are constant or, at least, are semi-continuous in such families, that is they can change only in "exceptional points" which form a family of smaller dimension. Perhaps the best known results in this direction are those of Gabriel [Gab] and Knörrer [Kn]. Gabriel proved that finite representation type is an open condition for finite dimensional algebras ("fat points"), while Knörrer showed that the number of parameters for modules of prescribed rank is semi-continuous in families of commutative Cohen-Macaulay rings of Krull dimension 1 ("curve singularities"). In [DG 2] Knörrer's theorem was used to show that the unimodal singularities of type $T_{p q}$ are of tame Cohen-Macaulay type.

Unfortunately, the arguments of [Kn] do not work in the non-commutative case. The aim of this paper is to refine them in such a way that they could be applied to non-commutative Cohen-Macaulay algebras, too. For this purpose we introduce the notion of "dense subrings" which seems rather technical but, nevertheless, useful. It enables the construction of "almost versal" families of modules for a given algebra (cf. Theorem 3.5) and the definition of the "number of parameters". Just as in the commutative case, it is important that the bases of these "almost versal" families are projective varieties. Once having this, we are able to prove an analogue of Knörrer's theorem (cf. Theorem 4.9) and a certain variant (cf. Theorem 4.11) which turns out to be useful, for instance, to extend the tameness criterion for commutative algebras |DG 2 to the case of characteristic 2. The semicontinuity implies, in particular, that the set of so-called "wild algebras" in any family is a countable union of closed subsets. A very exciting problem is whether it is actually closed, hence whether the set of tame algebras is open. However, Theorem 4.9, together with the results of DG 2], imply that tame is indeed an open property for curve singularities (commutative one-dimensional Cohen-Macaulay rings).

An analogous procedure leads to the semicontinuity of the number of parameters in other cases, like representations of finite dimensional algebras or elements of finite dimensional bimodules.

Though we do not consider here the problem of constructing moduli spaces for Cohen-Macaulay modules (cf. [GP]), we may rephrase the semicontinuity theorem by saying that the dimension of the moduli space for such modules of prescribed rank varies upper semicontinuosly in flat families of Cohen-Macaulay algebras. Likewise, the semicontinuity in other cases (finite-dimensional algebras or bimodules) may be also understood as semicontinuity of the dimension of the corresponding moduli spaces of representations under deformations of the algebra or bimodule.

Unfortunately, our results are, just as all known results till now, not sufficient to prove the "tame is open condition"-conjecture. Nevertheless, the semicontinuity theorem as well as the construction of "almost versal" families of modules are not restricted to tame algebras. They have a potentially broader field of applications to classification problems in representation theory. They are a particularly powerful tool if, for a given algebra, the deformation theory of this algebra is sufficiently known and the classification problem for the deformed algebras is easier to solve or 
even known. The great success of this approach in the commutative case is, of course, also due to the fact that the deformation theory of singularities is a highly developed field. We hope that this paper stimulates further research in the deformation theory for non-commutative Cohen-Macaulay algebras. 


\section{Dense subalgebras}

Definition 1.1 Let $B$ be a subring of a ring $A$. Call $B$ dense in $A$ if any simple $A$-module $U$ is also simple as $B-E n d_{A} U$-bimodule.

\section{Examples:}

1. If $A$ is commutative, then $\operatorname{End}_{A} U=A / A n n U$, so any subring $B$ is dense in A.

2. We can take $A=M_{2}(\mathbb{R})$ the ring of $2 \times 2$ real matrices and $B=\mathbb{C}$ (or even $B=\mathbb{Q}(i))$, naturally embedded in is $A$. More generally, let $k$ be some field, $L, K$ and $B$ be its extensions such that $L=K B$ (a composite) and $(L: K)=n$. Then we have a natural embedding $L \rightarrow A=M_{n}(K)$, thus also $B$ is a subalgebra of $A$ and $B$ is dense in $A$.

Lemma 1.2 Let $D$ be a division algebra over an infinite field $k, A=M_{n}(D)$ and $B \subset A$ a dense subalgebra. Consider $W=M_{n \times m}(D)$ as $A$-module and let $V \subset W$ be a $B$-submodule such that $A V=W$. Suppose that $m=n q+r$ with $0 \leq r<n$. Then there exists an automorphism $\sigma$ of $W$ such that $\sigma(V)$ contains the matrices

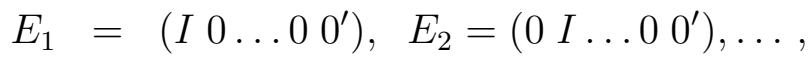

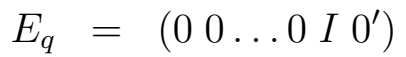

and a matrix of the form $\left(Y_{1} Y_{2} \ldots Y_{q} Y^{\prime}\right)$.

Here $I$ denotes the $n \times n$ unit matrix,

0 denotes the $n \times n$ zero matrix,

$0^{\prime}$ denotes the $n \times r$ zero matrix,

$Y_{1}, Y_{2}, \ldots, Y_{q}$ are some $n \times n$ matrices and $Y^{\prime}$ is an $n \times r$ matrix of rank $r$ (of course, if $r=0$, then $0^{\prime}$ and $Y^{\prime}$ are empty, so in this case $V$ contains an $A$-basis of $W \simeq q A)$.

Proof: Use induction on $m$. First prove the claim in the case $m \leq n$. Choose a matrix $X \in V$ of maximal possible rank, say $d$. Then we must show that $d=$ $m$. Suppose that $d<m$. Denote by $\bar{X}_{1}, \bar{X}_{2}, \ldots, \bar{X}_{m}$ the columns of $X$ and let $\bar{X}_{1}, \bar{X}_{2}, \ldots, \bar{X}_{d}$ be linear independent. Then there exists an automorphism $\sigma$ of $W$ such that the last $(m-d)$ columns of $\sigma(X)$ are zero. So we may suppose that $\bar{X}_{d+1}=\ldots=\bar{X}_{m}=\overline{0}$. Note that $W \simeq m U$ where $U=n D$ is the only simple $A$-module. As $d<n, \bar{X}_{1}, \bar{X}_{2}, \ldots, \bar{X}_{d}$ do not span $U$ over $D$. Denote by $V^{\prime}$ the projection of $V$ onto the $(d+1)$-st component of $W$ (i.e. $V^{\prime} \subset U$ consists of the first $d+1$ columns of the matrix from $V)$. As $A V=W$, we have $A V^{\prime}=U$, so $V^{\prime} \neq 0$. But as $U$ is a simple $B$-D-bimodule, $V^{\prime} D=U$. Therefore, $V$ contains a matrix $Y$ such that its $(d+1)$-st column $\bar{Y}_{d+1}$ does not lie in $\left\langle\bar{X}_{1}, \bar{X}_{2}, \ldots, \bar{X}_{d}\right\rangle D$. Choose a $D$-basis of $U$ of the form $\left\{\bar{X}_{1}, \bar{X}_{2}, \ldots, \bar{X}_{d}, \bar{Y}_{d+1}, \bar{Z}_{d+2}, \ldots, \bar{Z}_{n}\right\}$ and let

$$
\bar{Y}_{i}=\sum_{j=1}^{d} \bar{X}_{i} \lambda_{i j}+\bar{Y}_{d+1} \lambda_{i, d+1}+\sum_{j=d+2}^{n} \bar{Z}_{j} \lambda_{i j} .
$$


Again, using an automorphism of $W$, we may suppose that $\lambda_{i, d+1}=0$ for $i \neq d+1$. But then $\operatorname{rank}(\gamma X+Y) \geq d+1$ for some $\gamma \in k$, which is a contradiction. Hence, $d=m$. In particular, if $m=n$, there exists an automorphism $\sigma$ of $W$, such that $\sigma(X)=I$. Thus, our claim is proved for $m \leq n$.

Suppose now that $m>n$ and consider the projection $V^{\prime}$ of $V$ onto $W^{\prime}=n U$, the first $n$ components of $W$ (that is the first $n$ columns of each matrix $X \in W$ ). As we have proved, there exists an automorphism $\sigma^{\prime}$ of $W^{\prime}$ such that $\sigma^{\prime}\left(V^{\prime}\right) \ni I$. We can extend $\sigma^{\prime}$ to $W$ and thus suppose that $V$ contains a matrix $X$ of the form $\left(I X^{\prime}\right)$. Again using an automorphism, we obtain that $X^{\prime}=0$, that is $X=E_{1}$. Now consider the projection $V^{\prime \prime}$ of $V$ onto $W^{\prime \prime}=(m-n) U$, the last components of $W$. Using induction, we may also suppose that the claim is valid for $V^{\prime \prime}$, thus $V$ contains the matrices of the form:

$$
\begin{aligned}
& \left(\begin{array}{llllll}
X_{2} & I & 0 & \ldots & 0 & 0^{\prime}
\end{array}\right), \quad\left(\begin{array}{llllll}
X_{3} & 0 & I & \ldots & 0 & 0^{\prime}
\end{array}\right), \ldots,
\end{aligned}
$$

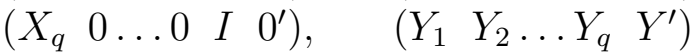

with $\operatorname{rank}\left(Y^{\prime}\right)=r$. But then, again using an automorphism of $W$, we can make $X_{2}=X_{3}=\ldots=X_{q}=0$, q.e.d.

Corollary 1.3 Let $B \subset A$ be a dense subring. Suppose that $A / \operatorname{rad} A$ is an artinian ring containing an infinite field $k$ in its centre and, moreover, $k \subset B /(B \cap \operatorname{rad} A)$ (for example $B$ and $A$ are $k$-algebras). Let $V \subset n A$ be a $B$-submodule such that $A V=n A$. Then $V$ contains an $A$-basis of $n A$.

Proof: Of course, we may replace $A$ by $A / \operatorname{rad} A$, so suppose that $A=\prod_{i=1}^{s} A_{i}$ with $A_{i}$ simple artinian. Put $B_{i}=p r_{i} B, V_{i}=p r_{i} V, p r_{i}$ being the projection from $A$ onto $A_{i}$. Then $B_{i}$ is dense in $A_{i}$ and $A_{i} V_{i}=n A_{i}$. Lemma 1.2 implies that each $V_{i}$ contains an $A_{i}$-basis $\left\{\bar{e}_{i j} \mid j=1, \ldots, n\right\}$ of $n A_{i}$. Let $e_{i j} \in V$ be such elements that $p r_{i} e_{i j}=$ $\bar{e}_{i j}(j=1, \ldots, n ; i=1, \ldots, s)$. Consider in $V$ the elements $e_{j}\left(\lambda_{1}, \ldots, \lambda_{s}\right)=$ $\sum_{i=1}^{s} \lambda_{i} e_{i j}$ where $\lambda_{1}, \ldots, \lambda_{s} \in k$. The sets $T_{i}=\left\{\left(\lambda_{1}, \ldots, \lambda_{s}\right) \mid p r_{i} e_{j}\left(\lambda_{1}, \ldots, \lambda_{s}\right)\right.$ form a basis of $\left.n A_{i}\right\}$, are Zariski-open in $k^{s}$ and non-empty. As $k$ is infinite, their intersection is also non-empty.

But if $\left(\lambda_{1}, \ldots, \lambda_{s}\right)$ lies in this intersection, then $\left\{e_{j}\left(\lambda_{1}, \ldots, \lambda_{s}\right) \mid j=1,2, \ldots, n\right\}$ is a basis of $n A$, q.e.d.

Definition 1.4 Let $D$ be a skewfield, $A=M_{n}(D), U=n D$ the simple $A-$ module and

$$
\mathcal{F}: U=U_{0} \supset U_{1} \supset \ldots \supset U_{s}=\{0\}
$$

a flag of $D$-subspaces in $U$. Put $A(\mathcal{F})=\left\{a \in A \mid a U_{i} \subset U_{i}\right.$ for all $\left.i=0,1, \ldots, m\right\}$ and call $A(\mathcal{F})$ a flag subalgebra in $A$. If $A=\prod_{i} A_{i}$ with $A_{i}$ simple artinian, call a flag subalgebra of $A$ any subalgebra $A^{\prime}$ of the form $A^{\prime}=\Pi_{i} A_{i}^{\prime}$ where $A_{i}^{\prime}$ is a flag subalgebra in $A_{i}$ for each $i$.

Lemma 1.5 Let $A$ be a semi-simple artinian ring, $A(\mathcal{F})$ a flag subalgebra in $A$ and $B$ a subring of $A(\mathcal{F})$. Then there exists a flag subalgebra $A^{\prime}$ such that $B \subset A^{\prime} \subset$ $A(\mathcal{F})$ and $B$ is dense in $A^{\prime}$. 
Proof: Obviously, we may suppose $A$ to be simple. Then take a maximal $B-$ invariant flag $\mathcal{F}^{\prime} \supset \mathcal{F}$ and put $A^{\prime}=A\left(\mathcal{F}^{\prime}\right)$.

Proposition 1.6 Let $A$ be an algebra over a separably closed field $k$, such that $A / \mathrm{rad} A$ is finite-dimensional over $k, B$ a dense subalgebra of $A$ and $K$ a separably generated extension of $k$. Then $B \otimes_{k} K$ is also dense in $A \otimes_{k} K$.

Proof: Of course, we may suppose $A$ to be simple finite-dimensional, that is $A=$ $M_{n}(F)$ for some skewfield $F$. As $k$ has no separable extensions, $F$ is really a field [DK], so it is a pure inseparable extension of $k$. But then $F \otimes_{k} K$ is again a field, hence $A \otimes_{k} K$ is simple. Its only simple module is $U \otimes_{k} K$, where $U$ is the simple $A$-module and $F \otimes_{k} K$ is its endormorphism ring. But the same observation shows that for any simple $B-F$-bimodule $V$ (e.g. for $U$ ) the tensor product $V \otimes_{k} K$ remains simple, q.e.d. 


\section{Cohen-Macaulay Algebras}

We consider here one-dimensional Cohen-Macaulay algebras (not necessarily commutative), also known as orders in semi-simple algebras.

Definition 2.1 Call a ring $\Lambda$ a CM-algebra (more precisely, 1-dimensional, analytically reduced Cohen-Macaulay algebra) if it satisfies the following conditions:

(1) $\Lambda$ is an algebra over a one-dimensional local, commutative, noetherian ring $R$, which is a finitely generated and torsion-free $R$-module.

(2) The completion $\widehat{\Lambda}$ of $\Lambda$ in the $\mathfrak{m}$-adic topology, where $\mathfrak{m}$ is the maximal ideal of $R$, contains no nilpotent ideals.

It follows from (2) that, in this case, $R$ is a Cohen-Macaulay ring and $\Lambda$ is a maximal Cohen-Macaulay $R$-module.

In particular, the $\mathfrak{m}$-adic completion $\widehat{R}$ of $R$ has no nilpotent elements. Denote by $Q$ (respectively $\widehat{Q}$ ) the total ring of fractions of $R$ (respectively $\widehat{R}$ ). Then both $Q$ and $\widehat{Q}$ are finite products of fields and $Q \Lambda=Q \otimes_{R} \Lambda\left(\widehat{Q} \Lambda=\widehat{Q} \otimes_{R} \Lambda=\widehat{Q} \otimes_{\widehat{R}} \widehat{\Lambda}\right)$ is a semi-simple artinian $Q$-algebra (respectively $\widehat{Q}$-algebra). If $\Gamma$ is a subring of $Q \Lambda$, containing $\Lambda$ and being finitely generated as $\Lambda$-module (or, equivalently, as $R$-module), call it an overring of $\Lambda$. Of course, any such overring is also a CMalgebra. If $\Lambda$ has no proper overrings, call it a maximal CM-algebra. It is known (cf., e.g., [D1]) that, under condition (1), condition (2) is equivalent to the existence of maximal overrings of $\Lambda$. More precisely, under conditions (1) and (2), the overrings of $\Lambda$ satisfy the ascending chain conditions and any two maximal overrings of $\Lambda$ are conjugate in $Q \Lambda$.

Let $\Lambda$ be a CM-algebra. We call a $\Lambda$-module $M$ a $\Lambda$-lattice (or a CohenMacaulay $\Lambda$-module) provided it is a maximal Cohen-Macaulay $R$-module. Denote by $\operatorname{CM}(\Lambda)$ the category of all $\Lambda$-lattices. Any such lattice $M$ embeds naturally into the finitely generated $Q \Lambda$-module $Q M=Q \otimes_{R} M$. So, if $\Gamma$ is an overring of $\Lambda$, the $\Gamma$-module $\Gamma M \subset Q M$ is well-defined.

The following assertions are rather well-known (for the case when $R$ is a discrete valuation ring, cf. Rog; the proofs in the general situation are the same).

Proposition 2.2 (a) Any maximal CM-algebra $\Lambda$ is hereditary (that is gl.dim $\Lambda=1$ or, equivalently, any $\Lambda$-lattice is projective).

(b) Let $\Lambda$ be a maximal $C M$-algebra and $A$ any flag subalgebra of $\Lambda / \operatorname{rad} \Lambda$ (cf. Definition 1.4). Then the preimage of $A$ in $\Lambda$ is hereditary and any hereditary $C M$-algebra can be obtained in this way.

Corollary 2.3 Let $\Omega$ be a hereditary (e.g. maximal) overring of a CM-algebra $\Lambda$. Then there exists a hereditary overring $\Omega^{\prime}$ such that $\Lambda \subset \Omega^{\prime} \subset \Omega$ and $\Lambda$ is dense in $\Omega^{\prime}$. 
Proposition 2.4 Suppose that the residue field $k=R / \mathfrak{m}$ is infinite. Let $\Gamma$ be an overring of $\Lambda$ such that $\Lambda$ is dense in $\Gamma$ and $M$ be a Cohen-Macaulay $\Lambda$-module such that $\Gamma M \simeq n \Gamma$. Then $M$ is isomorphic to a module $M^{\prime}$ such that $n \Lambda \subset M^{\prime} \subset n \Gamma$.

Proof: We may suppose that $M \subset n \Gamma$. By Corollary 1.3 it contains a basis of $n \Gamma$. Then there exists an automorphism $\sigma$ of $n \Gamma$ which maps this basis to the standard one, namely $(1,0, \ldots, 0),(0,1,0, \ldots, 0), \ldots,(0, \ldots, 0,1)$. Therefore, $M^{\prime}=\sigma(M) \supset n \Lambda$.

Proposition 2.5 If $M, M^{\prime} \subset n \Gamma$ are $\Lambda$-submodules such that $\Gamma M=\Gamma M^{\prime}=n \Gamma$ (e.g. $M$ and $M^{\prime}$ contain $n \Lambda$ ), then $M \simeq M^{\prime}$ if and only if there exists an automorphism $\sigma \in \operatorname{Aut}(n \Gamma)$ such that $\sigma(M)=M^{\prime}$.

The proof is evident.

Definition 2.6 For any Cohen-Macaulay $\Lambda$-module $M$ denote $\ell(M)$ the length of the $Q \Lambda$-module $Q M$ and call it the rational length of $M$.

Remark: If $\Gamma$ is an overring of $\Lambda$ and $M$ is a Cohen-Macaulay $\Gamma$-module, the rational length of $M$ does not depend on whether we consider $M$ as a $\Lambda_{-}$or as a $\Gamma$-module. On the other hand, we have to distinguish between $\ell(M)$ and $\ell(\widehat{M})$ where $\widehat{M}$ is the $\mathfrak{m}$-adic completion of $M$.

Recall some connections between Cohen-Macaulay modules and their completions. The proofs can be found in [CR or [Rog] for the case when $R$ is a discrete valuation ring, and they are also valid in the general situation.

Proposition 2.7 (a) $M \simeq N$ if and only if $\widehat{M} \simeq \widehat{N}$.

(b) If $N$ is a Cohen-Macaulay $\widehat{\Lambda}-$ module such that $Q N \simeq Q \widehat{N}^{\prime}$ for some CohenMacaulay $\Lambda$-module $N^{\prime}$, then there exists a Cohen-Macaulay $\Lambda$-module $N^{\prime \prime}$ such that $N \simeq \widehat{N}^{\prime \prime}$.

(c) If $\widehat{N}$ is isomorphic to a direct summand of $\widehat{M}$, then $N$ is isomorphic to a direct summand of $M$.

In the next section we shall use the following simple result.

Proposition 2.8 Let $P$ be a projective $\Lambda$-module. Then there exists a projective $\Lambda$-module $P^{\prime}$ such that $P \oplus P^{\prime}$ is free of rank $r \leq \operatorname{dim}_{k}(P / \operatorname{rad} P)$ where $k=R / \mathfrak{m}$.

Proof: Due to Proposition 2.7, we may suppose that $R$ is complete, thus the Krull-Schmidt theorem holds for modules. Let $\Lambda \simeq \oplus_{i=1}^{s} n_{i} P_{i}$, where all $P_{i}$ are indecomposable and pairwise non-isomorphic. Then $P \simeq \oplus_{i=1}^{s} m_{i} P_{i}$ for some $m_{i}$.

Take $r$ the least integer such that $r n_{i} \geq m_{i}$ for all $i$. Then $r \Lambda \simeq P \oplus P^{\prime}$ for $P^{\prime}=\oplus_{i=1}^{s}\left(r n_{i}-m_{i}\right) P_{i}$. As $\operatorname{dim}_{k}(P / \operatorname{rad} P)=\sum_{i=1}^{s} m_{i} \operatorname{dim}_{k}\left(P_{i} / \operatorname{rad} P_{i}\right) \geq m_{i}$, one obtains $r \leq \operatorname{dim}_{k}(P / \operatorname{rad} P)$, q.e.d.

Remark: Obviously, $\operatorname{dim}_{k}(P / \operatorname{rad} P) \leq \ell(\widehat{P})$, so the last number can also serve as an upper bound for $r$. 


\section{$3 \quad$ Families of Modules}

From now on we suppose that our rings are algebras over the field $k=R / \mathfrak{m}$, where $R$ is, as in the preceding paragraph, a Cohen-Macaulay ring.

Definition 3.1 Let $X$ be a $k$-scheme, $\mathcal{O}_{X}=\mathcal{O}$ its structure sheaf, $\Lambda$ a $C M$-algebra (1-dimensional and analytically reduced) and $\mathcal{M}$ a coherent sheaf on $X$ of $\Lambda \otimes_{k} \mathcal{O}_{-}$ modules. Call $\mathcal{M}$ a family of Cohen-Macaulay $\Lambda$-modules on $X$ if the following conditions hold:

(1) $\mathcal{M}$ is $R$-torsion free.

(2) $\mathcal{M}$ is $\mathcal{O}$-flat.

(3) For each point $x \in X, \mathcal{M}(x)=\mathcal{M} \otimes_{\mathcal{O}} k(x)$ is a Cohen-Macaulay $\Lambda(x)$-module, where $\Lambda(x)=\Lambda \otimes_{k} k(x)$.

It is easy to see that, under conditions (1) and (2), condition (3) is equivalent to:

(3') For every non-zero divisor $a \in R$, the sheaf $\mathcal{M} / a \mathcal{M}$ is also $\mathcal{O}$-flat.

We are going to construct some "almost universal" families. Let $\Gamma$ be an overring of $\Lambda$ (cf. §2) and fix some positive integers $n$ and $d$. Put $\Phi=\Gamma / \Lambda$ and consider the Grassmannian $G r=\operatorname{Gr}(n \Phi, d)$, that is the variety of subspaces of codimension $d$ in $n \Phi$. Recall that for every $k$-scheme $X$ the morphisms $X \rightarrow G r$ are in 1-1 correspondence with $\mathcal{O}$-factormodules of $n \Phi \otimes_{k} \mathcal{O}_{X}$ which are locally free of rank $d$ Mum. Consider the subvariety $B=B(n, d ; \Lambda, \Gamma)$ of $\operatorname{Gr}(n \Phi, d)$ consisting of all $\Lambda$ submodules of $n \Phi$. In other words, the morphisms $X \rightarrow B$ are in 1-1 correspondence with $\Lambda \otimes_{k} \mathcal{O}_{X}$-factormodules of $n \Phi \otimes_{k} \mathcal{O}_{X}$ which are locally free over $\mathcal{O}_{X}$ of rank $d$. Evidently it is a closed subscheme of $G r$. Denote by $\mathcal{F}=\mathcal{F}(n, d ; \Lambda, \Gamma)$ the preimage in $n \Gamma \otimes_{k} \mathcal{O}_{B}$ of the canonical locally free sheaf of corank $d$ on $B$. As $\left(n \Gamma \otimes_{k} \mathcal{O}_{B}\right) / \mathcal{F}$ is flat over $\mathcal{O}_{B}$, one can see that $\mathcal{F}$ is really a family of Cohen-Macaulay $\Lambda$-modules on $B$ having the following universal property (cf. [GP]).

Proposition 3.2 For any family of Cohen-Macaulay $\Lambda$-modules $\mathcal{M}$ on a scheme $X$ such that $n \Lambda \otimes_{k} \mathcal{O}_{X} \subset \mathcal{M} \subset n \Gamma \otimes_{k} \mathcal{O}_{X}$ and $\left(n \Gamma \otimes_{k} \mathcal{O}_{X}\right) / \mathcal{M}$ is locally free over $\mathcal{O}_{X}$ of rank $d$, there exists a unique morphism $\varphi: X \rightarrow B$ such that $\mathcal{M}=\varphi^{*}(\mathcal{F})$.

Definition 3.3 Call the families satisfying the conditions of Proposition 3.9 sandwiched families with respect to $\Gamma$ of rank $n$ and codimension d. In particular, when $X=$ Spec $k$, we have sandwiched modules (with respect to $\Gamma$ ).

From now on we suppose the ground field $k$ to be algebraically closed. We are going to show that the sandwiched families are, in some sense "almost versal", that is any other families can be stably glued from finitely many sandwiched families. Taking into account Corollary 2.3, this follows from the following result. 
Theorem 3.4 Let $\Gamma$ be a hereditary overring of $\Lambda$ such that $\Lambda$ is dense in $\Gamma$. Then, given a family $\mathcal{M}$ of Cohen-Macaulay $\Lambda$-modules on a reduced $k$-scheme $X$, there exists a descending chain of closed subschemes $X=X_{0} \supset X_{1} \supset X_{2} \supset \cdots \supset X_{m}=\emptyset$, a set of morphisms $\left\{\varphi_{i}: Y_{i} \longrightarrow B\left(n_{i}, d_{i} ; \Lambda, \Gamma\right) \mid i=1, \ldots, m\right\}$ and a set of projective $\Gamma$-modules $\left\{P_{i} \mid i=1, \ldots, m\right\}$ such that $\mathcal{M}_{Y_{i}} \oplus\left(P_{i} \otimes_{k} \mathcal{O}_{Y_{i}}\right) \simeq \varphi_{i}^{*} \mathcal{F}\left(n_{i}, d_{i} ; \Delta, \Gamma\right)$ where $Y_{i}=X_{i-1} \backslash X_{i}$ and $n_{i} \leq \hat{\ell}(\mathcal{M})=\ell(\widehat{\mathcal{M}}(x))$ for an arbitrary closed point $x \in X$.

Indeed, we shall establish a more general result, when $\Gamma$ is not necessarily hereditary, but $\Gamma \mathcal{M}$ is flat over $\Gamma \otimes_{k} \mathcal{O}_{X}$. If $\Gamma$ is hereditary, the last condition becomes superfluous. Since $\Gamma \mathcal{M} / \mathcal{M}$ is $\mathcal{O}_{X}$-coherent, there exists an open dense subset $U \subseteq X$, on which $\Gamma \mathcal{M} / \mathcal{M}$ is flat over $\mathcal{O}$. Then, as $\Gamma$ is hereditary, it follows from [CE (Theorem 2.8) that $\Gamma \mathcal{M}$ is also $\Gamma \otimes_{k} \mathcal{O}$-flat. Moreover, the function $x \mapsto \operatorname{dim}_{k(x)} \Gamma \mathcal{M}(x) / \operatorname{rad} \Gamma \mathcal{M}(x)$ takes its maximum in some closed point of $X$ and it does not exceed $\hat{\ell}(\mathcal{M})$. Hence, we need only to establish the following fact:

Proposition 3.5 Let $\Gamma$ be an overring of $\Lambda$ such that $\Lambda$ is dense in $\Gamma$. Let $\mathcal{M}$ be a family of Cohen-Macaulay $\Lambda$-modules on a reduced $k$-scheme $X$ such that $\Gamma \mathcal{M}$ is flat over $\Gamma \otimes_{k} \mathcal{O}$. Then there exists an open subscheme $Y \subset X$, a projective $\Gamma-$ module $P$ and a morphism $\varphi: Y \rightarrow B(n, d ; \Lambda, \Gamma)$ for some integers $n$ and $d$ such that the restriction on $Y$ of the family $\mathcal{M} \oplus\left(P \otimes_{k} \mathcal{O}\right)$ is isomorphic to $\varphi^{*} \mathcal{F}(n, d ; \Lambda, \Gamma)$. Moreover, we can choose $n \leq \max _{g} \ell(\mathcal{M}(g))$ where $g$ runs through minimal points of $X$ (that is generic points of its irreducible components).

Proof: Of course, we may suppose that $X$ is irreducible. Let $g \in X$ be its generic point. Consider the $\Gamma$-module $\Gamma \mathcal{M}(g)$. It is finitely generated and flat over $\Gamma(g)$, hence projective $\mathrm{Bou}$, (Ch. I, §2, Ex. 15). By Proposition 2.8, there exists a projective $\Gamma(g)$-module $P^{\prime}$ such that $\Gamma \mathcal{M}(g) \oplus P^{\prime} \simeq n \Gamma(g)$ and we can choose $n \leq \operatorname{dim}_{k(g)}(\Gamma \mathcal{M}(g) / \operatorname{rad} \Gamma \mathcal{M}(g))$. If we move to the completions, there is a $1-$ 1 -correspondence between projective and semi-simple $\widehat{\Gamma}$-modules and the same is valid for $\widehat{\Gamma}(g)$-modules. But as $k$ is separably closed and $k(g)$ separably generated over $k$, we have seen in the proof of Proposition 1.6 that any simple $\widehat{\Gamma}(g)$-module is of the form $U \otimes_{k} k(g)$ for some simple $\widehat{\Gamma}$-module $U$. Hence, the same is true for projectives, so $\widehat{P}^{\prime} \simeq \widehat{P} \otimes_{k} k(g)$ for some projective $\widehat{\Gamma}$-module $\widehat{P}$. But Proposition 2.7 implies then that $\widehat{P}$ is really a completion of some projective $\Gamma$-module $P$, whence $P^{\prime} \simeq P \otimes_{k} k(g)$.

Replacing $\mathcal{M}$ by $\mathcal{M} \oplus\left(P \otimes_{k} \mathcal{O}\right)$, we may now suppose that $\Gamma \mathcal{M}(g) \simeq n \Gamma(g)$. But $\Lambda(g)$ is dense in $\Gamma(g)$ by Proposition 1.6, so we may suppose, using Corollary 1.3, that $\mathcal{M}(g)$ contains a basis of $n \Gamma(g)$. By Proposition 2.4, $\mathcal{M}(g)$ is isomorphic to a submodule of $n \Gamma(g)$ containing $n \Lambda(g)$. So let $n \Lambda(g) \subset \mathcal{M}(g) \subset n \Gamma(g)$. Then the same is true on an open subset $Y \subset X$, that is $n \Lambda \otimes \mathcal{O}_{Y} \subset \mathcal{M}_{Y} \subset n \Gamma \otimes \mathcal{O}_{Y}$. Shrinking $Y$, we may also suppose that $\left(n \Gamma \otimes \mathcal{O}_{Y}\right) / \mathcal{M}_{Y}$ is locally free of some rank $d$ (over $\mathcal{O}_{Y}$ ) and it remains to use Proposition 3.2.

An obvious iteration gives us the necessary generalization of Theorem 3.4:

Corollary 3.6 Under the conditions of Proposition 3.5 there exists a descending chain of closed subschemes $X=X_{0} \supset X_{1} \supset X_{2} \supset \ldots \supset X_{n}=\emptyset$, a set of morphisms 
$\left\{\varphi_{i}: Y_{i} \rightarrow B\left(n_{i}, d_{i}, \Lambda, \Gamma\right) \mid i=1,2, \ldots, n\right\}$ and a set of projective $\Gamma$-modules $\left\{P_{i} \mid i=\right.$ $1,2, \ldots, n\}$ such that $\mathcal{M}_{Y_{i}} \oplus\left(P_{i} \otimes_{k} \mathcal{O}_{Y_{i}}\right) \simeq \varphi_{i}^{*} \mathcal{F}\left(n_{i}, d_{i} ; \Lambda, \Gamma\right)$ where $Y_{i}=X_{i-1} \backslash X_{i}$ and $n_{i} \leq \max \left\{\operatorname{dim}_{k(x)}(\Gamma \mathcal{M}(x) / \operatorname{rad} \Gamma \mathcal{M}(x)) \mid x \in X\right\}$.

Fix now a CM-algebra $\Lambda$ and an overring $\Gamma$. Let $B=B(n, d ; \Lambda, \Gamma)$ and $\mathcal{F}=$ $\mathcal{F}(n, d ; \Lambda, \Gamma)$. Choose a two-sided $\Gamma$-ideal $I \subset \operatorname{rad} \Lambda$ of finite codimension (over $k$ ) and put $F=\Gamma / I ; \bar{\Lambda}=\Lambda / I$. We can identify $\operatorname{Gr}(n \Phi, d)$ with the closed subscheme of $\operatorname{Gr}(n F, d)$ consisting of all subspaces $V$ containing $n \bar{\Lambda}$. Then $B$ also becomes a closed subvariety of $\operatorname{Gr}(n F, d)$. We shall consider the elements of $n F$ as rows of length $n$ with entries from $F$ and identify $\operatorname{Aut}(n F)$ with the full linear group $G L(n, F)=G$ acting on $n F$ according to the rule $g \cdot v=v g^{-1}$. Then Proposition 2.5 implies that two subspaces $V, V^{\prime} \in B$ correspond to isomorphic sandwiched modules if and only if there exists an element $g \in G$ such that $g \cdot V=V^{\prime}$.

Considering the elements of $n F$ as the rows of $n \times n$ matrices, we can identify $n V$ with a subspace in $M_{n}(F)$. Then we obtain the following:

Proposition 3.7 Let $V \in B, g \in G$. Then $g \cdot V \in B$ if and only if $g \in G \cap n V$. Hence, $G \cdot V \cap B=(G \cap n V) \cdot V \simeq(G \cap n V) / S t V$, where $S t V=\{g \in G \mid g V=V\}$.

As $G$ is open in $M_{n}(F), G \cap n V$ is open in $n V$, hence $\operatorname{dim}(G \cap n V)=\operatorname{dim} n V=$ $n(\gamma n-d)$ where $\gamma=\operatorname{dim} F$. Therefore, $\operatorname{dim}(G \cdot V \cap B)=n(\gamma n-d)-\operatorname{dim} S t V$, whence:

Corollary 3.8 For each integer $i$ the set $B_{i}=\{V \in B \mid \operatorname{dim}(G \cdot V \cap B) \leq i\}$ is closed in $B$.

Put

$$
\operatorname{par}(n, d ; \Lambda, \Gamma)=\max _{i}\left(\operatorname{dim} B_{i}-i\right)
$$

and

$$
\operatorname{par}(n ; \Lambda, \Gamma)=\max _{d} \operatorname{par}(n, d ; \Lambda, \Gamma) .
$$

Intuitively, $\operatorname{par}(n, d ; \Lambda, \Gamma)$ is the number of independent parameters defining the isomorphism classes of sandwiched $\Lambda$-modules of rank $n$ and codimension $d$ with respect to $\Gamma$. Corollary 3.6 evidently implies the following result.

Corollary 3.9 Under the conditions of Proposition 3.5, for any closed point $x \in X$ the set $\left\{y \in X \mid \mathcal{M}(y) \simeq \mathcal{M}(x) \otimes_{k} k(y)\right\}$ is constructible (that is a finite union of locally closed subsets of $X)$ and its dimension is bigger or equal to dimX$\operatorname{par}(\widehat{\ell}(\mathcal{M}) ; \Delta, \Gamma)$.

In particular, this assertion is true for any family of Cohen-Macaulay $\Lambda$-modules if we take for $\Gamma$ an hereditary overring of $\Lambda$ such that $\Lambda$ is dense in $\Gamma$ (which always exists, cf. Corollary 2.3).

Corollary 3.10 Let $\Gamma$ be any overring of $\Lambda$ and $\Omega$ a hereditary overring of $\Lambda$ such that $\Lambda$ is dense in $\Omega$. Put $\ell_{0}=\ell(\widehat{\Lambda})$. Then $\operatorname{par}(n, \Gamma) \leq \operatorname{par}\left(\ell_{0} n, \Omega\right)$ for all $n$. 
Of course, if $\Gamma \subset \Gamma^{\prime}$ are two overrings of $\Lambda$ and $\operatorname{dim}_{k}\left(\Gamma^{\prime} / \Gamma\right)=c$, then $\operatorname{par}(n, d ; \Lambda, \Gamma) \leq$ $\operatorname{par}\left(n, c+d ; \Lambda, \Gamma^{\prime}\right)$, whence $\operatorname{par}(n ; \Lambda, \Gamma) \leq \operatorname{par}\left(n ; \Lambda, \Gamma^{\prime}\right)$. Put

$$
b(n, \Lambda)=\max \{\operatorname{par}(n ; \Lambda, \Gamma)\}
$$

where $\Gamma$ runs through all overrings of $\Lambda$ (we have actually to look only for maximal ones). Let also $p(n, \Lambda)$ denote the maximal value of $\operatorname{dim} X-\operatorname{dim}\{y \in X \mid \mathcal{M}(y) \simeq$ $\left.\mathcal{M}(x) \otimes_{k} k(y)\right\}$ taken for all families $\mathcal{M}$ with all possible bases $X$ and for all closed points $x \in X$.

Corollary 3.11 Let $\ell_{0}=\ell(\widehat{\Lambda})$. Then

$$
b(n, \Lambda) \leq p(n, \Lambda) \leq b\left(n \ell_{0}, \Lambda\right) .
$$




\section{Families of algebras}

Now we formulate and prove the semicontinuity statements in two variants: for "familes of algebras" (Theorems 4.7 and 4.9) and for "families of generators" (Theorem 4.11).

Again $k$ denotes an algebraically closed field.

Definition 4.1 Let $C$ be a reduced algebraic curve over $k, \Lambda$ a coherent sheaf of $\mathcal{O}_{C^{-}}$ algebras, containing no nilpotent ideals and such that for every point $p \in C, \Lambda_{p}$ is maximal Cohen-Macaulay $\mathcal{O}_{C, p}-$ module. Then we call $\Lambda$ a sheaf of CM-algebras or just a $\mathbf{C M}-$ algebra on $\mathbf{C}$.

If $\Gamma \supset \Lambda$ is another CM-algebra on $C$ and, for each $p \in C, \Gamma_{p}$ is overring of $\Lambda_{p}$, call $\Gamma$ an overring of $\Lambda$.

Proposition 4.2 If $\Lambda$ is a CM-algebra on a curve $C$, then, for every point $p \in C$, $\Lambda_{p}$ is a CM-algebra (in the sense of Definition 2.1) and, moreover, for almost all points $\Lambda_{p}$ is maximal.

Proof: We only need to prove that $\widehat{\Lambda}_{p}$ contains no nilpotent ideal. According to [D1], this is equivalent to the existence of a maximal overring of $\Lambda_{p}$. Denote by $Z$ the centre of $\Lambda_{p}$. It is a localization of a finitely generated $k$-algbra, hence, its algebraic closure $\bar{Z}$ in the total quotient ring $Q$ is a finitely generated $Z$-module (cf. [Bou], Ch.V. §3.2). As before, we consider $\Lambda_{p}$ embedded in $Q \Lambda_{p}=Q \otimes_{Z} \Lambda_{p}$. Therefore, $\bar{Z} \Lambda_{p} \subset Q \Lambda_{p}$ is well-defined. But now $Q \Lambda_{p}$ is a central, semi-simple, hence, separable $Q Z$-algebra, so $\bar{Z} \Lambda_{p}$ has a maximal overring (cf. [CR ). Moreover, as $\bar{Z}_{p}=Z_{p}$ for almost all $p \in C$ and $\bar{Z} \Lambda_{p}$ is maximal for almost all $p$, the same is true also for $\Lambda_{p}$, q.e.d.

Call $\Lambda$ hereditary if all $\Lambda_{p}$ are hereditary (note that, for a general point $g \in C$, $\Lambda_{g} \simeq Q \Lambda$ is semi-simple). It is well-known (cf. [CR]) that one-dimensional CMalgebras can be defined locally:

Proposition 4.3 Let $\Lambda$ be a CM-algebra on a curve $C$ and suppose that for each closed point $p \in C$ an overring $\Gamma(p) \supset \Lambda_{p}$ is given such that $\Gamma(p)=\Lambda_{p}$ for almost all $p$. Then there exists an overring $\Gamma \supset \Lambda$ such that $\Gamma_{p}=\Gamma(p)$ for all $p$.

Corollary 4.4 There exists a hereditary overring $\Omega \supset \Lambda$ such that $\Lambda_{p}$ is dense in $\Omega_{p}$ for each $p \in C$.

Let now $\Gamma$ be any overring of $\Lambda$. As $\Gamma_{p}=\Lambda_{p}$ for almost all $p$, the sum

$$
\operatorname{par}(n ; \Lambda, \Gamma)=\sum_{p \in C} \operatorname{par}\left(n ; \Lambda_{p}, \Gamma_{p}\right)
$$

is well-defined. 
Definition 4.5 Let $f: Y \rightarrow X$ be a morphism of $k$-schemes and $\mathcal{L}$ be a coherent sheaf of $\mathcal{O}_{Y}$-algebras. Call $(\mathcal{L}, f)=(\mathcal{L}, f: Y \rightarrow X)$ a family of CM-algebras with the base $X$ provided the following conditions hold:

(1) $f$ is flat and $f_{*}(\mathcal{L})$ is flat $\mathcal{O}_{X}$-module.

(2) $Y(x)=f^{-1}(x)$ is a reduced algebraic curve for each $x \in X$.

(3) $\mathcal{L}(x)$ is a CM-algebra on $Y(x)$ for each $x \in X$.

Definition 4.6 Let $(\mathcal{L}, f: Y \rightarrow X)$ be a family of $C M$-algebras with base $X$. $A$ family of overrings of $(\mathcal{L}, f)$ is a family $\left(\mathcal{L}^{\prime}, f\right)$ (with the same $f$ ) such that $\mathcal{L}^{\prime} \supset \mathcal{L}, f_{*}\left(\mathcal{L}^{\prime} / \mathcal{L}\right)$ is $\mathcal{O}_{X}$ flat and, for each $x \in X, \mathcal{L}^{\prime}(x)$ is an overring of $\mathcal{L}(x)$.

Given a family of overrings $\mathcal{L}^{\prime} \supset \mathcal{L}$, we can define the functions on $X$ :

$$
\begin{aligned}
\operatorname{par}(x, n, d) & :=\operatorname{par}\left(x, n, d ; \mathcal{L}, \mathcal{L}^{\prime}\right)=\operatorname{par}\left(n, d ; \mathcal{L}(x), \mathcal{L}^{\prime}(x)\right) \\
\operatorname{par}(x, n) & :=\operatorname{par}\left(x, n ; \mathcal{L}, \mathcal{L}^{\prime}\right)=\operatorname{par}\left(n ; \mathcal{L}(x), \mathcal{L}^{\prime}(x)\right)
\end{aligned}
$$

Theorem 4.7 The functions $\operatorname{par}(x, n, d)$ and $\operatorname{par}(x, n)$ are upper-semicontinuous, that is for each integer $i$ and for any $k$-scheme $X$ the sets $X_{i}(d)=\{x \in$ $X \mid \operatorname{par}(x, n, d) \geq i\}$ and $X_{i}=\{x \in X \mid \operatorname{par}(x, n) \geq i\}$ are closed in $X$.

Proof: As $X_{i}=\cup_{d} X_{i}(d)$ and since this union is finite, we only need to prove that $X_{i}(d)$ is closed. Moreover, we may suppose that $X$ is a smooth curve. Let $\mathcal{N}=\mathcal{L}^{\prime} / \mathcal{L}$. Consider the relative Grassmannian $\operatorname{Gr}(n \mathcal{N}, d) \rightarrow X$ and its closed subscheme (over $X) \mathcal{B}(n, d)$ consisting of $\mathcal{L}$-submodules. Let $\mathcal{J}$ be the biggest twosided $\mathcal{L}^{\prime}$-ideal contained in $\mathcal{L}$. Then it is easy to see that $\mathcal{L} / \mathcal{J}$ is torsion-free over $\mathcal{O}_{X}$, hence, flat. Thus, $\mathcal{L}^{\prime} / \mathcal{J}$ is also flat over $\mathcal{O}_{X}$. As in the proof of Proposition 3.7, identify $\operatorname{Gr}(n \mathcal{N}, d)$ with the closed subscheme of $\operatorname{Gr}\left(n \overline{\mathcal{L}^{\prime}}, d\right)$, where $\overline{\mathcal{L}}^{\prime}=\mathcal{L}^{\prime} / \mathcal{J}$, and consider the group scheme over $X, G L\left(n, \overline{\mathcal{L}}^{\prime}\right)$ acting on the last Grassmannian. The same observations as in the proof of Proposition 3.7 shows that $\mathcal{B}_{j}=\{v \in \mathcal{B}(n, d)$ $\operatorname{dim} S t v \geq j\}$ is closed in $\mathcal{B}$. As $\mathcal{B}$ is proper over $X$, its projection $Z_{j}$ is also closed. But, by definition $X_{i}=\cup_{j} X_{i j}$, where $X_{i j}=\left\{x \in Z_{j} \mid \operatorname{dim} \mathcal{B}_{j}(x) \geq i+j\right\}$ are closed, q.e.d.

Remark: Suppose that the base of the family $(\mathcal{L}, f)$ is a smooth curve and both $\mathcal{L}$ and $\mathcal{L}^{\prime}$ are Cohen-Macaulay $\mathcal{O}_{Y}$-modules. Then $\left(\mathcal{L}^{\prime}, f\right)$ is a family of overrings as it follows from $\mathrm{BG}$ (Example 3.2.5). Moreover, in this case $\mathcal{O}_{Y}$ is Cohen-Macaulay itself and $\operatorname{dim} Y=2$. Hence, we are able to construct Cohen-Macaulay $\mathcal{O}_{Y}$-modules locally as in the following lemma.

Lemma 4.8 Suppose that $Y$ is a reduced 2-dimensional Cohen-Macaulay variety. Let $\mathcal{M}$ be a Cohen-Macaulay $\mathcal{O}_{Y}$-module, $\left\{y_{1}, y_{2}, \ldots, y_{m}\right\}$ a set of points of $Y$ of codimension 1 and $N\left(y_{i}\right)$ a finitely generated $\mathcal{O}_{Y, y_{i}}-$ submodule in $\mathcal{Q} \mathcal{M}$ where $\mathcal{Q}$ is the total quotient ring of $\mathcal{O}$. Then there exists the Cohen-Macaulay submodule $\mathcal{N} \subset \mathcal{Q} \mathcal{M}$ such that $\mathcal{N}_{y_{i}}=N\left(y_{i}\right)$ and $\mathcal{N}_{y}=\mathcal{M}_{y}$ for all points y of codimension 1 , distinct from all $y_{i}$. 
Proof: One can easily construct $\mathcal{N}$ with prescribed localizations as in Bou (VII 4.3). Moreover, we may suppose that $\mathcal{N}=\cap_{\text {codim } y=1} \mathcal{N}_{y}$. But then $\mathcal{N}$ is CohenMacaulay.

We can now prove the main result of this paper. Recall that

$$
b(n, x):=b(n, \mathcal{L}(x))=\max \{\operatorname{par}(n ; \mathcal{L}(x), \Gamma\}
$$

is the maximum number of independent parameters of isomorphism classes of sandwiched $\mathcal{L}(x)$-modules of rank $n$, which can be thought of as the dimension of the "moduli space" of $\mathcal{L}(x)-\mathrm{CM}-$ modules of rank $n$.

Theorem 4.9 The function $b(n, x)=b(n, \mathcal{L}(x))$ is upper semi-continuous.

Proof: Again we may suppose that $X$ is a smooth curve. Let $g \in X$ be the generic point of $X$ and $\Lambda=\mathcal{L}(g)$. Find an overring $\Omega \supset \Lambda$ such that $b(n, \Lambda)=\operatorname{par}(n, \Lambda, \Omega)$. Using Lemma 4.8, we can construct a family of overrings $\mathcal{L}^{\prime} \supset \mathcal{L}$ with $\mathcal{L}^{\prime}(g)=\Omega$. As $b(x) \geq \operatorname{par}\left(n, \mathcal{L}(x), \mathcal{L}^{\prime}(x)\right)$ for every $x \in X$, it follows from Proposition 4.7 that the set $\{x \in X \mid b(x) \geq b(g)\}$ is closed. This, of course, proves the theorem.

Corollary 4.10 For any family of $C M$-algebras $(\mathcal{L}, f: Y \rightarrow X)$, the set $W(\mathcal{L})=$ $\{x \in X \mid \mathcal{L}(x)$ is wild $\}$ is a countable union of closed subsets of $X$.

(For the definition of tame and wild CM-algebras cf. [DG 1]).

The proof of this corollary follows from Theorem 4.8 just in the same way as it followed in the commutative case from Knörrer's theorem (cf. [DG 2], Corollary $4.2)$.

Now we consider onother version of the semicontinuity theorem, where algebras are given by parametrized families of generators. Namely, let $X$ be an algebraic $k-$ scheme, $\mathcal{L}$ a family of $\mathrm{CM}$-algebras with the base $X$ and $\mathcal{I}$ an ideal of $\mathcal{L}$ such that $\mathcal{L} / \mathcal{I}$ is a locally free $\mathcal{O}_{X}$-module of finite rank, that is it corresponds to a vector bundle $\pi: F \rightarrow X$. The fibres $F(x)$ of $F$ are then finite-dimensional $k(x)$-algebras. Suppose given an algebraic $X$-scheme $f: Y \rightarrow X$ and a set of $X$-morphisms $\left\{\gamma_{i}: Y \rightarrow F \mid i=1,2, \ldots, m\right\}$ (equivalently $Y$-sections of $f^{*} F$ ).

For each point $y \in Y$ denote $A(y)$ the subalgebra of $F(f(y))$ generated by $\left\{\gamma_{1}(y), \gamma_{2}(y), \ldots, \gamma_{m}(y)\right\}$ and $\Lambda(y)$ the preimage of $A(y)$ in $\mathcal{L}(y)=\mathcal{L} \otimes_{\mathcal{O}_{X}} k(f(y))$. Then $\Lambda(y)$ is a CM-algebra, thus, given a family of overrings $\mathcal{L}^{\prime} \supset \mathcal{L}$, we may consider, as above, the functions on $Y$ :

$$
\begin{aligned}
p(n, d ; y) & =\operatorname{par}\left(n, d ; \Lambda(y), \mathcal{L}^{\prime}(y)\right) \\
p(n ; y) & =\operatorname{par}\left(n ; \Lambda(y), \mathcal{L}^{\prime}(y)\right) ; \\
b(n, y) & =b(n, \Lambda(y)) .
\end{aligned}
$$

Theorem 4.11 In the above situation, the functions $p(n, d ; y) ; p(n, y)$ and $b(n, y)$ are upper-semicontinuous on $Y$. 
Proof: Replacing $\mathcal{L}$ by $f^{*}(\mathcal{L})$, which is a family of $\mathrm{CM}$-algebras on $Y$, we may suppose that $X=Y$ and $f$ is the identity map. Moreover, we may also suppose $X$ to be a smooth curve. As the function $\operatorname{dim} A(y)$ is obviously upper semi-continuous on $Y$, there is an open subset $U \subset Y$ such that $\operatorname{dim} A(y)$ is constant and maximal possible on $U$. Put $d=\operatorname{dim} F(y)-\operatorname{dim} A(y)$. Then we obtain a section $\varphi: U \rightarrow$ $\operatorname{Gr}(d, F)$ such that $A(y)$ is the subspace of $F(y)$ corresponding to $\varphi(y)$ for each $y \in U$. But as $X$ is a smooth curve and $\operatorname{Gr}(d, F)$ is projective over $X, \varphi$ can be prolonged to a section $\bar{\varphi}: X \rightarrow \operatorname{Gr}(d, F)$ (it follows, for example, from [Ha], Proposition III.9.8).

Now $\bar{\varphi}$ gives rise to a subbundle $A^{\prime} \subset F$ of constant codimension $d$. Denote by $\Lambda^{\prime}$ its preimage in $\mathcal{L}$. Note that both conditions

$$
\text { " } A^{\prime}(x) \text { is a subalgebra of } F(x) \text { " and " } A^{\prime}(x) \supset A(x) \text { " }
$$

are evidently closed and hold on $U$. Thus they hold on $X$, that is $\Lambda^{\prime}(x)$ is a subalgebra of $\mathcal{L}(x)$ containing $\Lambda(x)$. As $\mathcal{L} / \Lambda^{\prime}$ is locally free of finite rank, $\Lambda^{\prime}$ is really a family of $\mathrm{CM}$-algebras on $X$. Hence, the functions $p^{\prime}(n, d ; x), p^{\prime}(n ; x)$ and $b^{\prime}(n, x)$ defined just as $p(n, d ; x), p(n ; x)$ and $b(n, x)$ but using $\Lambda^{\prime}(x)$ instead of $\Lambda(x)$ are upper semicontinuous. On the other hand we have inequalitites $p(n, d ; x) \geq p^{\prime}(n, d ; x), p(n, x) \geq p^{\prime}(n, x), b(n, x) \geq b^{\prime}(n, x)$ on $X$ and equality on $U$. Therefore, $p(n, d ; x), p(n ; x)$ and $b(n ; x)$ are also upper semicontinuous.

To show an application of Theorem 4.11, we extend the criteria of tameness, proved in [DG 2] for the case char $k \neq 2$, to all characteristics. In order to do this, we must first define the singularities $T_{p q}$ in positive characteristic, which are defined for char $k=0$ as factorrings

$$
k[[X, Y]] /\left(X^{p}+Y^{q}+\lambda X^{2} Y^{2}\right) .
$$

For our purpose it is more convenient to define them using their parametrization given by Schappert $\mathrm{Sch}$. Namely, let $\Lambda$ be a local commutative CM-algebra, $\Lambda_{0}$ its maximal overring. Then $\Lambda_{0}$ is a direct product of power series rings:

$$
\Lambda_{0} \simeq k\left[\left[t_{1}\right]\right] \times k\left[\left[t_{2}\right]\right] \times \cdots \times k\left[\left[t_{s}\right]\right]
$$

( $s$ is "the number of branches" of $\Lambda$ ). If $a \in \Lambda, a=\left(a_{1}, a_{2}, \ldots, a_{s}\right)$, with $a_{i} \in k\left[\left[t_{i}\right]\right]$, put $\boldsymbol{v}(a)=\left(v\left(a_{1}\right), \ldots, v\left(a_{2}\right)\right)$, where $v\left(a_{i}\right)$ denotes the usual valuation on the power series (in particular $v(0)=\infty$ ).

Call $\Lambda$ a plane curve singularity if its maximal ideal $\mathcal{M}$ is generated by two elements: $\mathcal{M}=(x, y)$. Define the (valuation) type of $\Lambda$ as the pair $(\boldsymbol{v}(x), \boldsymbol{v}(y))$.

Definition 4.12 Let $\Lambda$ be a plane curve singularity. We say that $\Lambda$ is of type $\boldsymbol{T}_{\boldsymbol{p q}}$, where $p, q \in \mathbb{N}, \frac{1}{p}+\frac{1}{q} \leq \frac{1}{2}$, if its valuation type is:

$$
\begin{array}{ll}
(2, p-2),(q-2,2) & \text { for } p, q \text { both odd, } \\
(1,1, p-2),\left(\infty, \frac{q}{2}-1,2\right) & \text { for } p \text { odd, } q \text { even, } \\
\left(1,1, \frac{p}{2}-1, \infty\right),\left(\frac{q}{2}-1, \infty, 1,1\right) & \text { for } p, q \text { both even. }
\end{array}
$$

By Sch this definition is equivalent to the equation $(*)$ if char $k=0$. 
The following theorem was proved in [DG 2] for char $k \neq 2$.

Let $\Lambda$ be a local commutative CM-algebra, $\Lambda_{0}=k\left[\left[t_{1}\right]\right] \times \cdots \times k\left[\left[t_{s}\right]\right]$ its maximal overring, $\boldsymbol{m}=\operatorname{rad} \Lambda$. Denote $t=\left(t_{1}, \ldots, t_{s}\right) \in \Lambda_{0} ; \Lambda^{\prime}=t \Lambda_{0}+\Lambda, \Lambda^{\prime \prime}=t \boldsymbol{m} \Lambda_{0}+\Lambda$ and $\Lambda_{e}^{\prime}=\Lambda^{\prime}+k e$, where $e \in \Lambda^{\prime}$ is an idempotent. For each overring $\Gamma \supset \Lambda^{\prime}$, let $\Gamma / \boldsymbol{m} \Gamma=$ $L_{1} \times \cdots \times L_{m}$, where $L_{i}$ are local algebras, $d_{i}=\operatorname{dim} L_{i}, \boldsymbol{d}(\Gamma)=\left(d_{1}, d_{2}, \ldots, d_{m}\right)$ and $d(\Gamma)=d_{1}+\cdots+d_{m}$ (the minimal number of generators of $\Gamma$ as $\Lambda$-module).

Theorem 4.13 If $\Lambda$ is of infinite Cohen-Macaulay type, the following conditions are equivalent:

(1) $\Lambda$ is tame.

(2) $\Lambda$ dominates a plane curve singularity of type $T_{p q}$ for some $p, q$ (that is, $\Lambda$ is isomorphic to an overring of $T_{p q}$ ).

(3) The following restrictions hold:

(a) $d\left(\Lambda_{0}\right) \leq 4$ and $\boldsymbol{d}\left(\Lambda_{0}\right) \notin\{(4),(3,1),(3)\}$,

(b) $d\left(\Lambda^{\prime}\right) \leq 3$ and $\boldsymbol{d}\left(\Lambda_{e}^{\prime}\right) \neq(3,1)$ for any idempotent $e$,

(c) if $d\left(\Lambda_{0}\right)=3$, then $d\left(\Lambda^{\prime \prime}\right) \leq 2$.

Proof: $(1) \Rightarrow(3)$ and $(3) \Rightarrow(2)$ were proved in DG 2 and their proofs did not use the restriction char $k \neq 2$. In order to prove $(2) \Rightarrow(1)$, again following [DG 2], note that the singularity $\Lambda$ of type $T_{p q}$ contains the $\Lambda_{0}$-ideal $I=b \Lambda_{0}$, where

$$
\begin{array}{ll}
b=\left(t_{1}^{p+1}, t_{2}^{q+1}\right) & \text { for } p, q \text { both odd; } \\
b=\left(t_{1}^{q / 2+1}, t_{2}^{q / 2+1}, t_{3}^{p+1}\right) & \text { for } p \text { odd, } q \text { even; } \\
b=\left(t_{1}^{q / 2+1}, t_{2}^{p / 2+1}, t_{3}^{q / 2+1}, t_{4}^{p / 2+1}\right) & \text { for } p, q \text { both even. }
\end{array}
$$

Consider now a new CM-algebra $\Lambda(\lambda), \lambda \in k$, containing $I$ and generated modulo $I$ by the following 3 elements:

$$
\begin{array}{ll}
(\lambda, 1) x,(1, \lambda) y, x y & \text { for } p, q \text { both odd, } \\
(1,1, \lambda) x,(\lambda, \lambda, 1) y, x y & \text { for } p \text { odd, } q \text { even, } \\
(1, \lambda, 1, \lambda) x,(\lambda, 1, \lambda, 1) y, x y & \text { for } p, q \text { both even. }
\end{array}
$$

If $(p, q) \notin\{(4,4),(3,6)\}$, one can easily check that $\Lambda(\lambda) \simeq \Lambda$ for $\lambda \neq 0$, while $\Lambda(0)$ is a singularity of type $P_{p q}$ in the terminology of [DG 2], that is generated modulo $I$ by the elements $x_{0}, y_{0}$ such that $\boldsymbol{v}\left(x_{0}\right), \boldsymbol{v}\left(y_{0}\right)$ are of the form:

$$
\begin{array}{ll}
(2, \infty),(\infty, 2) & \text { for } p, q \text { both odd, } \\
(1,1, \infty),(\infty, \infty, 2) & \text { for } p \text { odd, } q \text { even, } \\
(1,1, \infty, \infty),(\infty, \infty, 1,1) & \text { for } p, q \text { both even }
\end{array}
$$

Again, the calculations for $P_{p q}$ in $[\overline{\mathrm{DG}}$ 2] did not use the condition char $k \neq 2$. Hence, they are tame and Theorem 4.11 implies that $\Lambda$ is also tame. The calculation of Dieterich for the remaining case $(p, q)=(3,6)$ or $(p, q)=(4,4)$ (cf. [Di 1], Di 2 ) also did not use any conditions on characteristics. Thus, implication $(2) \Rightarrow(1)$ is completely proved. 


\section{Some analogues}

Here we give some examples of "almost versal families" and semicontinuity theorems for other situations in representation theory. As all the proofs are quite similar (and easier) to those of the preceding sections, we omit them and give only the final formulations of the results analogous to Theorems 3.4, 4.7 and 4.11. Although some of the corresponding semicontinuity theorems are known, we hope that the "unification" will be useful for these cases too. At least we give new proofs for them.

\section{Finite-dimensional algebras}

Here, let $A$ be a finite-dimensional algebra over an algebraically closed field $k$. A family of $\boldsymbol{A}$-modules parametrized by a $k$-scheme $X$ is a sheaf $\mathcal{M}$ of $A \otimes_{k} \mathcal{O}_{X^{-}}$ modules, which is coherent and flat over $\mathcal{O}_{X}$. To be in the frame of projective varieties, we can consider first the subvariety $B(P, I, d) \subset G r(P, d)$, where $P$ is a projective module over a finite-dimensional algebra $A, I$ an ideal of $A$ contained in the radical and $B(P, I, d)$ consists of the $A$-submodules $L \subset I P$. $G r(P, d)$ denotes the Grassmannian of $d$-codimensional subspaces of $P$. Then the canonical sheaf $\mathcal{F}=\mathcal{F}(P, I, d)$ on $B(P, I, d)$ is a family of $A$-modules and the following result holds.

Theorem 5.1 Let $A$ be a finite-dimensional $k$-algebra, $J=\operatorname{rad} A$ and $\mathcal{M}$ a family of $A$-modules parametrized by a reduced $k$-scheme $X$. Then there exists a descending chain of closed subschemes $X=X_{0} \supset X_{1} \supset X_{2} \supset \cdots \supset X_{m}=\emptyset$ and a set of morphisms $\left\{\varphi_{i}: Y_{i} \rightarrow B\left(P_{i}, J, d_{i}\right) \mid i=1, \ldots, m\right\}$ for some projective $A$-modules $P_{i}$ such that $\mathcal{M}_{Y_{i}} \simeq \varphi_{i}^{*} \mathcal{F}\left(P_{i}, J, d_{i}\right)$, where $Y_{i}=X_{i-1} \backslash X_{i}$. Moreover, if $r=\operatorname{rank} \mathcal{M}$ (as a locally free sheaf over $X$ ), then $\operatorname{dim} P_{i} \leq r p$, where $p$ is the maximal dimension of indecomposable projective $A$-modules.

The group $G=\operatorname{Aut}_{A} P$ acts on $B=B(P, I, d)$ and, as $I \subset \operatorname{rad} A$, we conclude that $\mathcal{F}(x) \simeq \mathcal{F}(y)$ if and only if $x$ and $y$ belong to the same $G$-orbit. The subsets $B_{i}=\{x \in B \mid \operatorname{dim}(G x) \leq i\}$ are obviously closed in $B$. Hence, we can define the number of parameters:

$$
\operatorname{par}(P, I, d ; A)=\max _{i}\left(\operatorname{dim} B_{i}-i\right)
$$

and

$$
\operatorname{par}(P, I ; A)=\max _{d} \operatorname{par}(P, I, d ;, A)
$$

In particular, put

$$
\operatorname{par}(n, d ; A)=\operatorname{par}(n A, \operatorname{rad} A, d ; A) \quad \text { and } \quad \operatorname{par}(n ; A)=\operatorname{par}(n A, \operatorname{rad} A ; A) .
$$

Just as for Cohen-Macaulay algebras, these numbers give upper bounds for the number of (independent) parameters of isomorphism classes of $A$-modules of rank $n$ in any family of $A$-modules. 
Consider now a family of algebras parametrized by a $k$-scheme $X$, that is a flat coherent sheaf of $\mathcal{O}_{X}$-algebras $\mathcal{A}$. Then we are able to define the following functions on $X$ :

$$
\begin{aligned}
\operatorname{par}(x, n, d) & =\operatorname{par}(n, d ; \mathcal{A}(x)), \\
\operatorname{par}(x, n) & =\operatorname{par}(n ; \mathcal{A}(x)) .
\end{aligned}
$$

Theorem 5.2 For each family of finite-dimensional $k$-algebras the functions $\operatorname{par}(x, n, d)$ and $\operatorname{par}(x, n)$ are upper-semicontinuous.

A version of this theorem was proved by Geiß Geil. Note also that Theorem 5.2 provides a new proof of Gabriel's theorem Gab that finite representation type is an open condition. This follows since the Brauer-Thrall conjectures are known to be true for finite dimensional algebras.

It is easy to generalize the last theorem to the situation where the algebras are given by "generators and relations". Namely, suppose we are given:

- a family $\mathcal{A}$ of finite-dimensional $k$-algebras over $X$;

- an algebraic $X$-scheme $f: Y \rightarrow X$;

- two sets of $X$-morphisms $\left\{\gamma_{i}: Y \rightarrow F \mid i=1, \ldots, m\right\}$ and $\left\{\rho_{j}: Y \rightarrow F \mid j=\right.$ $1, \ldots, r\}$, where $F$ is the vector bundle on $X$ corresponding to the locally free sheaf $\mathcal{A}$.

For any point $y \in Y$, denote by $I(y)$ the ideal in $F(f(y))$ generated by the set $\left\{\rho_{j}(y) \mid j=1, \ldots, r\right\}$ and by $A(y)$ the subalgebra of $F(f(y)) / I(y)$ generated by the classes $\left\{\gamma_{i}(y)+I(y) \mid i=1, \ldots, m\right\}$. Then we can define the functions on $Y$ :

$$
\begin{aligned}
p(y, n, d) & =\operatorname{par}(n, d ; A(y)), \\
p(y, n) & =\operatorname{par}(n ; A(y)) .
\end{aligned}
$$

Theorem 5.3 In the above situation the functions $p(y, n, d)$ and $p(y, n)$ are uppersemicontinuous on $Y$.

\section{Bimodules}

Consider now the categories of elements of finite-dimensional bimodules (in the sense of [D2], although we give here a somewhat different definition). Let $A$ be a finitedimensional $k$-algebra, where $k$ is again an algebraically closed field, and let $V$ be a finite-dimensional $A$-bimodule. The elements of $V$ are, by definition, those of the set $E l(V)=\bigsqcup_{P} V(P)$, where $P$ runs through all (finitely generated) projective $A$ modules and $V(P)=\operatorname{Hom}_{A}\left(P, V \otimes_{A} P\right)$. Two elements $u \in V(P)$ and $u^{\prime} \in V\left(P^{\prime}\right)$ are said to be isomorphic if there exists an isomorphism $p: P \rightarrow P^{\prime}$ such that 
$u^{\prime}=(1 \otimes p) u p^{-1}$. Indeed, in D2 only so-called disjoint bimodules were considered. The bimodule $V$ is said to be disjoint if $A=A_{1} \times A_{2}$ and $V A_{1}=A_{2} V=0$. In most applications in representations theory one needs only disjoint bimodules, but non-disjoint ones appear in various "reduction processes".

To remain in the category of projective varieties, it is convenient to change the problem slightly. Namely, call elements $u$ and $u^{\prime}$ (projectively) equivalent, if $u^{\prime}$ is isomorphic to $\lambda u$ for some non-zero $\lambda \in k$. Obviously, if the bimodule is disjoint, then equivalent elements are isomorphic, but in the non-disjoint case it is not always so.

Let $X$ be a $k$-scheme and $\mathcal{P}$ a flat coherent sheaf of $A_{X}$-modules, where $A_{X}=$ $A \otimes_{k} \mathcal{O}_{X}$. Put $V(\mathcal{P})=\mathcal{H} o m_{A_{X}}\left(\mathcal{P}, V \otimes_{A} \mathcal{P}\right)$. It is a locally free coherent sheaf of $\mathcal{O}_{X}$-modules. Hence, the corresponding projective bundle $\mathbb{P}_{\mathcal{P}}=\mathbb{P}_{X}(V(\mathcal{P}))$ over $X$ is defined (cf. [Ha ). A projective family (or simply family, as we do not consider other families here) of elements of the bimodule $V$ with base $X$ is, by definition, a section $\Phi: X \rightarrow \mathbb{P}_{\mathcal{P}}$ for some $\mathcal{P}$. Note that using projective families we need to consider projective equivalence instead of isomorphism and to exclude zero elements of the bimodule. But this does not essentially differ from the classification problem for the elements of bimodules up to isomorphism.

The "almost universal" families in this case are more or less evident. Indeed, put, for any projective $A$-module $P, B(P)=\mathbb{P}_{k}(V(P))$ and $\tilde{P}=P \otimes_{k} \mathcal{O}_{B(P)}$. Then $\mathbb{P}_{\tilde{P}} \simeq B \times B$, where $B=B(P)$ and the diagonal map $\Delta_{P}: B \rightarrow B \times B$ defines a family of elements of $V$ with the base $B$. The following result is almost obvious.

Theorem 5.4 Let $A$ be a finite-dimensional $k$-algebra, $V$ a finite-dimensional $A$ bimodule and and $\Phi: X \rightarrow \mathbb{P}_{\mathcal{P}}$ a (projective) family of elements of $V$. Then there exists a descending chain of closed subschemes $X=X_{0} \supset X_{1} \supset X_{2} \supset \cdots \supset X_{m}=\emptyset$ and a set of morphisms $\left\{\varphi_{i}: Y_{i} \rightarrow B\left(P_{i}\right) \mid i=1, \ldots, m\right\}$ for some projective $A$ modules $P_{i}$ such that $\mathcal{P}_{Y_{i}} \simeq P_{i} \otimes_{k} \mathcal{O}_{Y_{i}}$. Hence, the restriction of $\mathbb{P}_{\mathcal{P}}$ on $Y_{i}=X_{i-1} \backslash X_{i}$ can be identified with $Y_{i} \times B\left(P_{i}\right)$, and, under this identification, $\Phi_{Y_{i}}=1 \times \varphi_{i}$. Moreover, $\operatorname{dim} P_{i}=\operatorname{rank} \mathcal{P}$ for all $i$.

The group $G=$ Aut $_{A} P$ acts on $B=B(P)$ and its orbits are the classes of projective equivalence. Hence, we are again able to define the closed subsets $B_{i}=\{x \in$ $B \mid \operatorname{dim}(G x) \leq i\}$ and the number of parameters:

$$
\operatorname{par}(P ; A, V)=\max _{i}\left(\operatorname{dim} B_{i}-i\right)
$$

in particular

$$
\operatorname{par}(n ; A, V)=\operatorname{par}(n A ; A, V) .
$$

Now, given a family of algebras $\mathcal{A}$ with base $X$ and a family of bimodules, that is a coherent sheaf $\mathcal{V}$ of $\mathcal{A}$-bimodules, flat over $\mathcal{O}_{X}$, we can define the function on $X$ :

$$
\operatorname{par}(x, n)=\operatorname{par}(n ; \mathcal{A}(x), \mathcal{V}(x))
$$


Theorem 5.5 For each family of finite-dimensional $k$-algebras and bimodules the function $\operatorname{par}(x, n)$ is upper-semicontinuous.

Of course, one could easily give a version of the last theorem, where the algebras and bimodules are defined by generators and relations, but we leave this obvious generalization to the reader.

\section{Remark}

In particular, in both cases we can see that the set of wild algebras (or bimodules) in some family is again a countable union of closed subsets. It looks very likely that this set is even closed and, hence, that the set of tame algebras (or bimodules) is open. In order to prove it, one only needs to show that the set of tame algebras (bimodules) is really a countable union of constructible sets (cf. Gab).

If we consider families of commutative CM-algebras, then the set of tame algebras is indeed open. This can be derived from [DG 2] in two ways. The first is to apply the classification of [DG 2] and deformation theory of singularities: the set of singularities which are of finite CM-representation type or which are tame is open in any flat family of singularities. The second is to note that the strict respresentations over the free algebra $k\langle x, y\rangle$ constructed in DG 2 are of bounded rank. Hence, we can find a common constant $n$ such that a commutative CM-algebra $\Lambda$ is wild if and only if $p(n, \Lambda)>r n$, where $r$ is the rational length of $\Lambda$, which coincides in this case with the number of branches. As $r$ is obviously bounded in any family, we have now only to apply Theorem 4.9. 


\section{References}

[BG] Buchweitz, R.-O.; Greuel, G.-M.: The Milnor number and deformations of complex curve singularities. Inv. Math. 58 (1980), 241-281.

[Bou] Bourbaki, N.: Algèbre Commutative. Paris: Hermann 1964-1969. (Translation: Commutative Algebra. Paris \& Reading: Hermann and Addison-Wesley, 1972.) 1969.

[CE] Cartan, H.; Eilenberg, S.: Homological Algebra. Princeton Univ. Press, Princeton 1956.

[CR] Curtis, C.W.; Reiner, I.: Methods of representation theory, vol. I. New York: Wiley \& Sons, 1978.

[Di 1] Dieterich E.: Solution of a non-dommestic tame classification problem from integral representation theory of finite groups. Mem. Amer. Math. Soc. 450 , 1991.

[Di 2] Dieterich E.: Lattice categories over curve singularities with large conductor. Preprint 92-069, SFB 343, Universität Bielefeld, 1992.

[D1] Drozd, Y.A.: Existence of maximal orders. Matm. Zametki, 37 (1985) 313-316 ( = Math. Notes of the Acad. of Sci. of the USSR, 37 (1985), 177-178).

[D2] Drozd, Y.A.: Tame and wild matrix problems. In: Representations and Quadratic Forms. Kiev, 29-74, 1979. (Translation in: Amer. Math. Soc. Transl. 128, 31-55, 1986.)

[DG 1] Drozd, Y.A.; Greuel, G.-M.: Tame-wild dichotomy for Cohen-Macaulay modules. Math. Ann. 294, 387-394 (1992).

[DG 2] Drozd, Y.A.; Greuel, G.-M.: Cohen-Macaulay module type. Compositio Math. 89, 315-338 (1993).

[DK] Drozd, Y.A.; Kirichenko, V.V.: Finite dimensional algebras. Springer-Verlag 1994.

[Gab] Gabriel, P.: Finite representation type is open. In: Representations of Algebras, Ottawa 1974 (Lecture Notes in Math. 488), Springer, 1975.

[Gei] Geiß, C.: On degenerations of tame and wild algebras. Arch. Math. 64, 11-16 (1995).

[GP] Greuel, G.-M.; Pfister, G.: Moduli Spaces for Torsion free Modules on Curve Singularities I. J. Algebraic Geometry 2, 81-135 (1993).

[Ha] Hartshorne, R.: Algebraic Geometry. Springer-Verlag 1977.

[Kn] Knörrer, H.: Torsionsfreie Moduln bei Deformation von Kurvensingularitäten. In: Singularities, Representation of Algebras and Vector Bundles, Lambrecht 1985 (Eds.: Greuel, G.-M.; Trautmann, G.). Lecture Notes in Math., Vol. 1273, pp. 150-155. Berlin-Heidelberg-New York: Springer 1987.

[Mum] Mumford, D: Lectures on Curves on an Algebraic Surface. Ann. of Mathematics Studies 59. Princeton Univ. Press, Princeton 1966. 
[Rog] Roggenkamp, K.W.: Lattices over Orders, II. Lecture Notes in Math., 142, Springer, 1970.

[Sch] Schappert, A.: Kurvensingularitäten und Isomorphieklassen von Moduln, Dissertation. Univ. Kaiserslautern, 1990. 